\title{
Erratum to: Remifentanil for labor analgesia: a comprehensive review
}

\author{
Yayoi Ohashi $^{1} \cdot$ Leyla Baghirzada $^{2} \cdot$ Hiroyuki Sumikura $^{3} \cdot$ Mrinalini Balki $^{4}$
}

Published online: 21 October 2016

(C) Japanese Society of Anesthesiologists 2016

\section{Erratum to: J Anesth}

\section{DOI 10.1007/s00540-016-2233-y}

The original publication of the article contains few errors which are corrected in this erratum.

1. In page 2, the first sentence under the heading "Comparison of remifentanil with other intravenous opioids and epidural analgesia (Table 1)" should read, Douma [10] compared pain scores and observer sedation scores among 159 women receiving remifentanil, fentanyl, and meperidine for labor analgesia, demonstrating a significant decrease in mean (SD) visual analogue scale (VAS) pain scores in the remifentanil group [-3.2 (2.9) cm] compared to fentanyl $[-1.4(2.4) \mathrm{cm}$; $p<0.01]$ and meperidine groups $[-0.8(2.2) \mathrm{cm}$; $p<0.001]$ at $1 \mathrm{~h}$, followed by a return to the baseline over time.

2. In Table 1, the word "acidiosis" should be "acidosis" for "Neonatal side effects" of Owen [45].

The online version of the original article can be found under doi:10.1007/s00540-016-2233-y.

Yayoi Ohashi

Yayoi.Ohashi@health.wa.gov.au

1 Department of Anaesthesia, Fremantle Hospital and Health Service, Alma Street, Fremantle, WA 6160, Australia

2 Department of Anesthesiology, South Health Campus, University of Calgary, Calgary, Canada

3 Department of Anesthesiology, Juntendo University, Tokyo, Japan

4 Department of Anesthesia and Pain Management, Mount Sinai Hospital, University of Toronto, Toronto, Canada
The above mentioned corrections have been updated in original article also. 